ISAHP 1996, Vancouver, Canada, July 12-15, 1996

\title{
A CONSENSUS MAKING SUPPORT SYSTEM USING AHP IN COMBINATION WITH K.J METHOD AND RELATIONSHIP MATRIX
}

\author{
Naotaka Kato and Susumu Kunifuji \\ Information Science \\ Japan Advanced Institute of Science and Technology,Hokuriku, Japan \\ nkato@jaist.ac.jp/kuni@jaist.ac.jp
}

\begin{abstract}
We propose a computer assisted method of consensus making for cooperative group decision problem solving. The problem solving has a process which constructs appropriate evaluation structure interactively and chooses the optimal alternative plan rationally. However, in this problem solving, the problem is usually complicated because it contains some ill-structured elements and each participant in the group has his own sense of value which is different from the others. Additionally, both subjective and objective evaluations are often needed in order to solve the problem. These make the problem solving more complexed. Our major concern is to support the problem solving rationally by using distributed computer systems. We expect the problem can be solved effectively by integrating various techniques of creative thinking support, system engineering, group decision support and groupware. This paper describes a consensus making support method which uses AHP in combination with a creative thinking method and a relationship matrix associating subjective evaluation with objective evaluation. The implementation example is also given.
\end{abstract}

\section{Introduction}

This paper focuses on an integrated architecture of requirement acquisition, creative thinking, decisionmaking, and groupware system. So far, one of the authors has designed and implemented a knowledge acquisition support groupware GRAPE(GRoupware for Acquiring, Processing, and Evaluating knowledge)(H.Ueda and S.Kunifuji,1993) by combining appropriate system analysis methodologies with system modelling methodologies(i.e., ISM(J.N.Warfield,1974), Extended ISM(S.kunifuji and T.Takeshima,1979), Fuzzy Clustering(L.A.Zadeh,1971), AHP(Analytic Hierarchy Process)(T.L.Saaty, 1980), and so on.) We have also developed another different system(H.Nagata,1994) to support the judgement which has the rationality of decision makers. These systems are bottom-up-typed group DSSs and have convergent thinking support functions. However, they are not sufficient for constructing appropriate evaluation structure because they don't have functions which can correct evaluation structure interactively. In case of the insufficient structure, iterative correction of its structure is necessary. For this purpose, we expect that integrating creative thinking method which is composed of divergent thinking process and convergent thinking process into these DSSs is useful. In the past, there are some creative thinking methods existing in Japan. Especially, KJ(Kawakita Jiro) method(J.Kawakita,1975) is one of the most interesting creative thinking methods, which is composed of divergent thinking process and convergent thinking process. It is widely used for two reasons. One is due to the suitability for requirement acquisition and requirement analysis(N.Takeda, A.Shiomi, K.Kawai and H.Ohiwa,1993). The other is that some useful computer assisted tools such as KJ Editor(H.Ohiwa, K.Kawai and M.Koyama,1990) and Diagram Abductor(K.Sugiyama and K.Misue,1991) have been developed. 
Inspired by these methods and systems, we are now designing and implementing a new type of consensus making support system for group decision problem solving(N.Kato and S.Kunifuji,1995;S.Kunifuji, T.Tamura and N.Kato,1995). The characteristics of our system are as follows:

- Combination of a creative thinking method (i.e. KJ method) and a group decision support method.

- A new hybrid system with divergent thinking support functions and convergent thinking support functions.

- Bi-directional transformation between subjective evaluation and objective evaluation using relationship matrix.

- Two types of tradeoff analysis mechanisms implimented for consensus making.

This paper is organized as follows; first, the outline of KJ method is described. Next, we describe our concepts and our consensus making support system in detail. Finally, some conclusions are given.

\section{KJ Method}

The original KJ method contains the following basic procedures.

1. Label Making: Each label is often derived from Brainstorming.

2. Label Grouping: It consists of label collection, grouping, and naming. A group can be nested and each group is also named. This label grouping is useful for getting a new hypothesis.

3. Chart Making: To find the relation among groups and/or labels. The relation may be similar, opposite, cause-from, etc. The chart is called A-type of KJ method.

The step of label making is a divergent thinking process, and the other steps are convergent thinking processes. The whole information of the creative thinking activity is concentrated in the A-type chart which is obtained by KJ method. An example chart of user's requirement in software development is shown in Figure 4. The chart externalizes the results of the creative thinking of all participants. Using this chart, we can extract the common part of the recognition and the common sense of value. These make KJ method useful for recognizing the common part in the early stage of consensus making. Moreover, the chart obtained by $\mathrm{KJ}$ method can be used as the hierarchical evaluation structure of AHP directly. In traditional AHP, this structure is usually obtained by a kind of non-systematic or heuristic method. We expect that the idea of applying KJ method, which is a systematic method, to construct the evaluation structure of AHP will give a better result than using the traditional method. From the above reasons, we apply KJ method to construct the evaluation structure of AHP for consensus making.

\section{Concept of the Proposed System}

Our concept is depicted in Figure 1. A requirement of a participant depends on his sense of value and also his stand point on the occasion of consensus making. So, firstly, we introduce a point of view that is called a priority as the basic measure to show some differences of sense of value and some degrees of compromise. According to this, we suppose that a participant's requirement is composed of some requirement elements associated with weight values denoting the priority. Such a requirement is represented by a hierarchical tree structure. Hereafter, we will simply deal with the requirement as mentioned above.

Next, we transform the requirement in Person A's sense of value into the dimension of requirement 


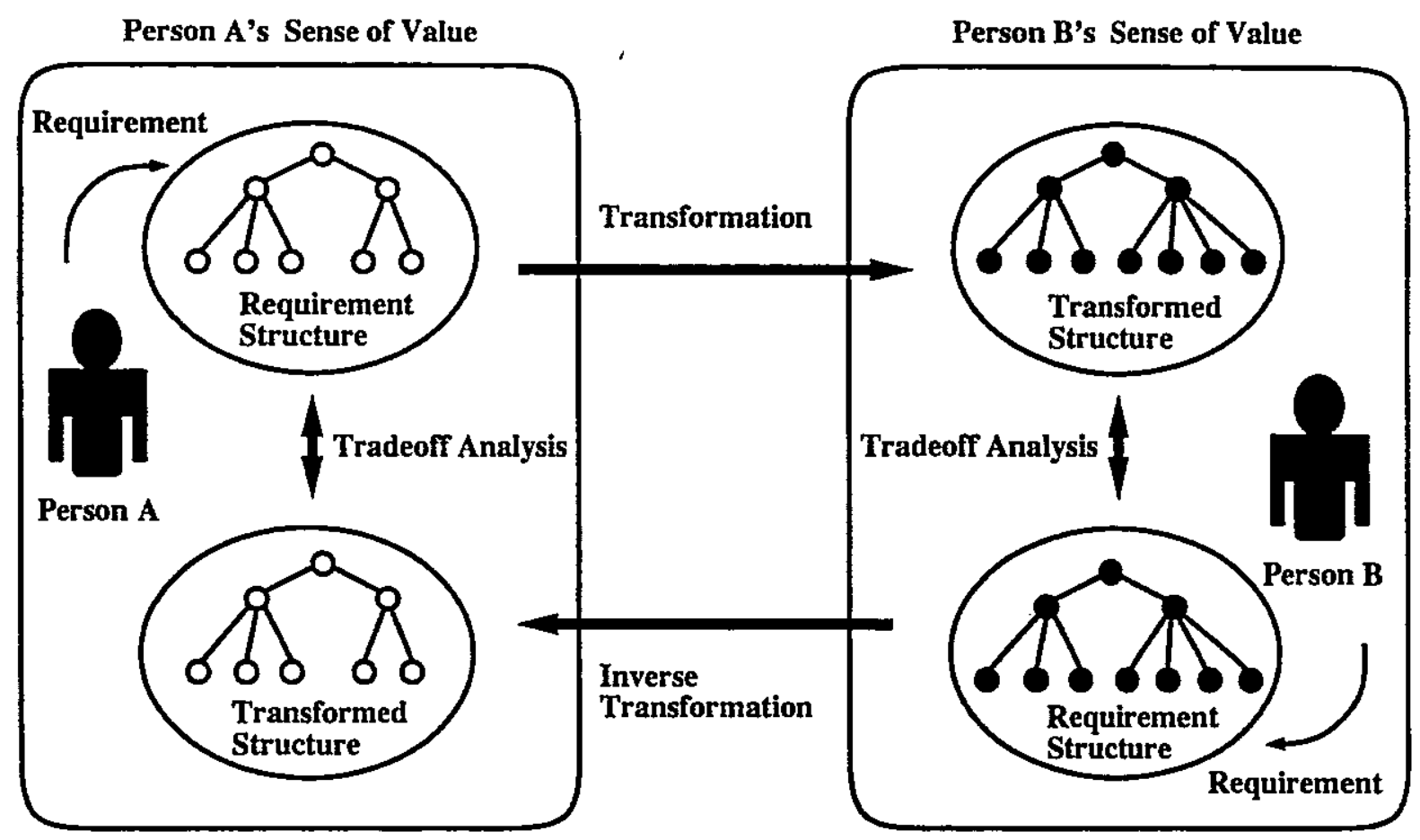

Figure 1: Concept of the proposed system

in Person B's sense of value. On the other hand, we transform the requirement in Person B's sense of value into the dimension of the requirement in Person $A$ 's sense of value vice versa. According to this, it is possible to do mutual adjusting of sense of value. In other words, the requirement in Person B's sense of value can be understood as the requirement which is possible to interpret in Person A's sense of value. The opposite matter is the same töo. Then, we believe that it is possible to support a consensus making by showing a mutual requirement each other. A relationship matrix is used for the process of these transformation and inverse transformation. We contrived this relationship matrix based on QDA(Quality Deployment Approach)(A.Ohmori,1994) which is known as the methodology of the quality control management.

By the way, the requirements of the participants are various. Especially, the opinion competition always exists among the participants. Some priorities have to be sacrified in order to carry out the other priority in some cases. So, appropriate value judgement of priority is necessary in such cases. Such a procedure is called tradeoff. To discovever the existerice of tradeoff which lurks among participants and to remove it efficiently is important in the consensus making process. Therefore, two kinds of tradeoff analysis mechanisms are implemented on our system to acquire the requirement of all participants and to support the consensus making, respectively. We define the tradeoff analysis as finding requirement element sets which have tradeoff relation and analyzing sensitivity for more effective consensus making.

\section{System Functions And Techniques}

The functional flow of our system is shown in Figure 2. It is composed of two function modules: requirement acquisition module and requirement analysis module.

- requirement acquisition module

It is composed of the following four sub-function modules: (1) requirement extraction, 


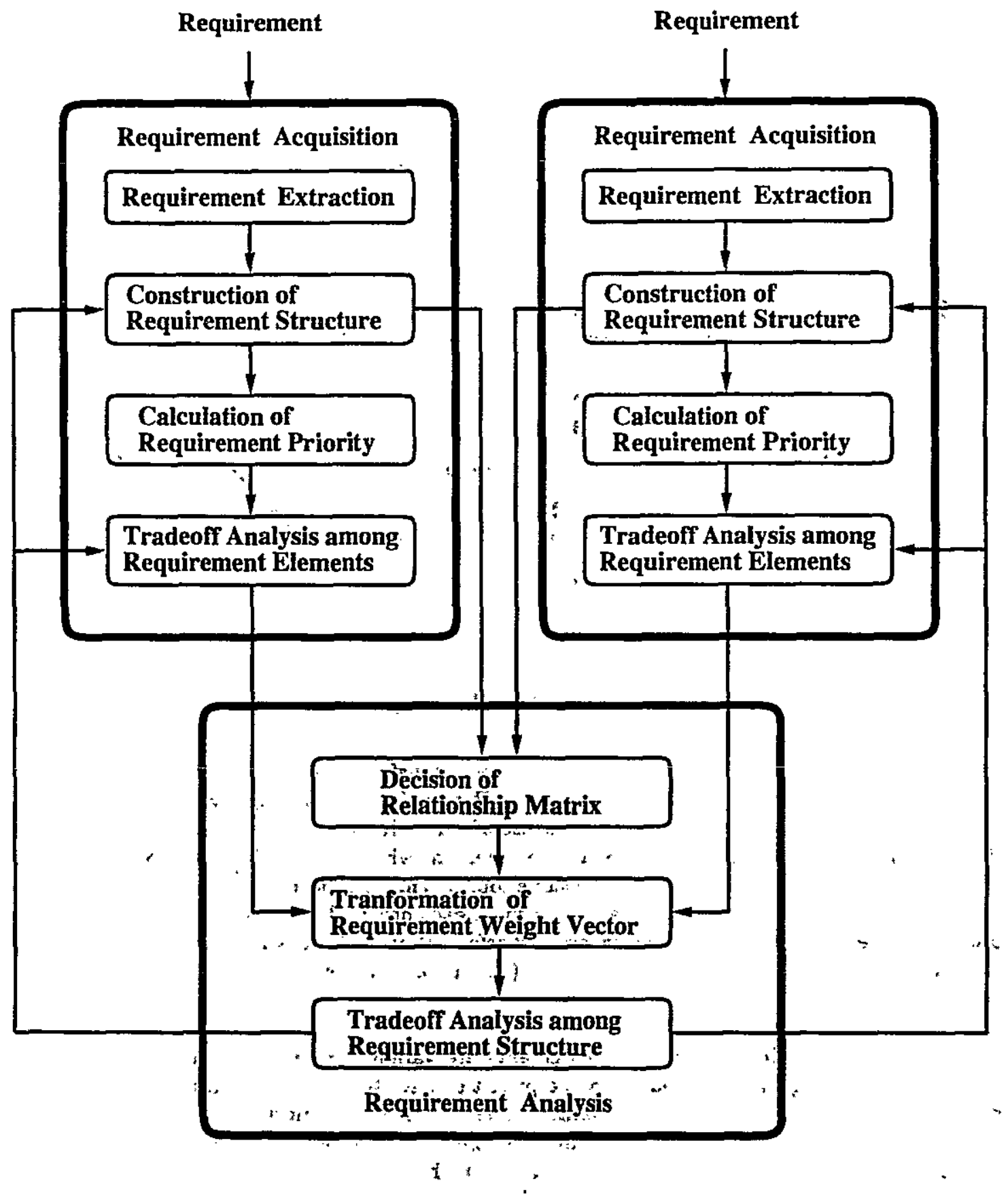

Figure 2: System function flow

construction of requirement structure, and (3) calculation of requirement priority, (4) tradeoff analysis among requirement elements. Here, Diagram Abductor can be used for module (1) and (2), which is a computer assisted tool for KJ method. ISM software can also be used for module (2).

- requirement analysis module

It is composed of the following four sub-function modules: (1) decision of relationship matrix, (2) transformation of requirement weight. vector, $1(3)$ inverse transformation of requirement weight vector, and (4) tradeoff analysis among requirement structure.

Each function module has an interactive operation environment and can execute as necessary on the 
$\mathrm{X}$ window system. For example, when noticing the existence of new requirement elements which should be added or ignored with progressing in the consensus making, the requirement structure can be appropriately corrected.

The detail of each function and technique is described in the order as follows.

\section{Requirement Acquisition}

The requirement acquisition is to extract requirement elements, to construct its structure, and to calculate priorities among these elements. As the result, requirements are embodied in the form of a set of the requirement elements associated with weight values denoting the priority and represented by a hierarchical tree structure.

\section{Requirement Extraction}

First, the tangible or latent requirement of the participants are collected in the form of the candid primitive word labels(for example, easiness, simplicity, portability, flexibility, and so on). Brainstorming is often used to collect the labels.

\section{Making of Requirement Structure}

We use KJ method to form the requirement structure which is composed of a set of the concise linguistic expression. To improve the efficiency of KJ label making, if necessary, it is possible to pick up KJ labels from the group collected in the past. By the similar procedure, we acquire the requirement of all of each participants. Incidentally, we construct the requirement structure using $\mathrm{KJ}$ method in principle, and ISM method is also available. This method is effective when the consciousness of participants to the problem and the knowledge level to the object knowledge are high.

\section{Calculation of Requirement Priority}

Generally, each requirement element differs in the measure and,"moreover, it has a subjective characteristic. 'We use AHP as the method of suiting priority calculation among such elements. AHP is a method of calculating ratio measure values with the consistency from doing each pair comparison among the elements and the values, assumed to be relative weights, denote the priority. Hereafter, we will deal with these weights as the priority of requirement elements.

As thè weight, AHP defines the eigen vector $\omega=\left(\omega_{1}, \omega_{2}, \ldots, \omega_{n}\right)$ of the maximum eigen value $\lambda_{\max }$ obtained from the pair comparison matrix $\mathrm{A}$ in formula(1);

$$
A=\left[a_{i j}\right]
$$

where $a_{i j}$ is a pair comparison value

$$
a_{i i}=1, a_{j i}=1 / a_{i j}, a_{i j}>0(1 \leq i \leq n, 1 \leq j \leq n)
$$

A relation between $a_{i j}$ and $\omega$ is shown by formula (3) when the consistency of the pair comparison matrix is complete.

$$
a_{i j}=\omega_{i} / \omega_{j}
$$

However, the consistency is generally incomplete because a pair comparison value is subjectively judged and fixed. According to the theory of AHP, the degree of consistency is called consistency index and is shown using symbol C.I.. C.I. is given by the following formula(4).

$$
\text { C.I. }=\left(\lambda_{\max }-n\right) /(n-1)
$$


By the way, the following two points are the problems of AHP.

1. Pair comparative work becomes complex when the number of the comparative elements increases. Consequentiy, the mental load of the worker increases.

2. It is not usual to get mutual complete independency, which is a condition of applying AHP, among the requirement elements obtained by $\mathrm{KJ}$ method. In other words, the dependency often exists among the requirement elements. Generally, it is difficult to remove this dependency totally.

Therefore, we apply incomplete pairwise comparison method in AHP(Harker,P.T.,1987) to problem 1 and method of AHP using non-additive weight(H.Ichihashi and H.Tanaka,1987) to problem 2.

\section{Incomplete Pairwise Comparison Method in AHP}

This is the technique composing the total pair comparison matrix which has a consistency as a whole by complementing the part for there to be a consistency in which there is not confidence in a pair comparison or not to be understood by the information lack. There is an effect that the number of the pair comparison decreases by using this technique. And this effect can also diminish the load of the pair comparison work when the number of the comparative elements is increased.

\section{AHP Using Non-additive Weight}

As for the requirement structure, the original weight values of AHP change and the reverse phenomenon of the weight order sometimes happens when there is the dependency among the requirement elements or when some new requirement elements are added. This reason is that the weight values are defined as the additive measure which is normalized for the weight summation to become 1 .

Therefore, with applying this method, we can remove the occurrence of the reverse phenomenon of the weight order. We normalize the weight values for the weight maximum to become 1 after finding them in original AHP.

Tradeoff Analysis Among Requirement Elements

When there is a difference between the expectation (or dissatisfaction) and the weight values found by the above procedure, it is necessary to revise the weight values. Also, in the next step of consensus making, the weight values must be adjusted to advance the compromise process of the mutual requirement. To achive the above purpose, the tradeoff analysis among the requirement elements becomes necessary. We propose an efficient technique of tradeoff analysis. Our approach applies the sensitivity coefficients of the weight vector $\omega$ (the values calculated by differentiating the weight vector $\omega$ by the pair comparison value $a_{i j}$ )(See T.Masuda,1987.) By this method, we can support the strategies which revise any pair comparison value by referring to these calculated sensitivity coefficients. More precisely, we can choose the combination of the pair comparison, that makes the tradeoff operation work with the most effectiveness. Our system shows a user the candidates $a_{i j}$ orderly with respect to the sensitivity coefficient. The user can revise the pair comparison value $a_{i j}$ which the tradeoff effect seems to be high. (i.e., the sensitivity coefficient is high.) Repetitively, the weight is re-calculated and the consistency index will be checked again. 


\section{Requirement Analysis}

The requirement analysis is to analyze prorities of requirement elements among some requirements of the participants. This section describes a technique for transforming the requirement in one's sense of value into the dimension of requirement in the other's sense of value. Next, the reverse procedure is described. Finally, we show the way to analyze these priorities by the tradeoff analysis technique. For simplicity, we explain these techniques using an example in a case of product design.

\section{Decision of Relationship Matrix}

Generally, a user's sense of value is subjective and qualitative, whereas a developer's sense of value is objective and quantitative. For example, the user's requirements are somethings like easiness of viewing display, easiness of operation, high speed processing and so on. Whereas the developer's requirements are such things as menu operation function, learning function, high-speed calculation function and so on. Therefore, we suppose the relationship table which provides the strength of the relationship between the user's requirement and the developer's requirement (for example, see Figure 3 ).

We suppose that the user's requirement elements is set to the row of the table, and the developer's requirement elements is set to the column of the table. Next, we place the strength of the relationship by the symbol like $($ (strong), $O$ (middle), $\Delta$ (weak) in the table. Lastiy, a relationship matrix is made by giving five points to $\bigcirc$, three points to $O$, one point to $\triangle$, and 0 points to the others. In this way, the relationship matrix of two types of requirements is represented by the two dimensional matrix. This procedure is based on QDA method which is developed for the product quality management to reflect a customer requirement and is widely used for the mechanical product design mainly.

\section{Transformation of Requirement Weight Vector}

By using this relationship matrix, a user's requirement is transformed into the dimension of a developer's requirement. Suppose that a weight vector of the user's requirement is $u$, a re-

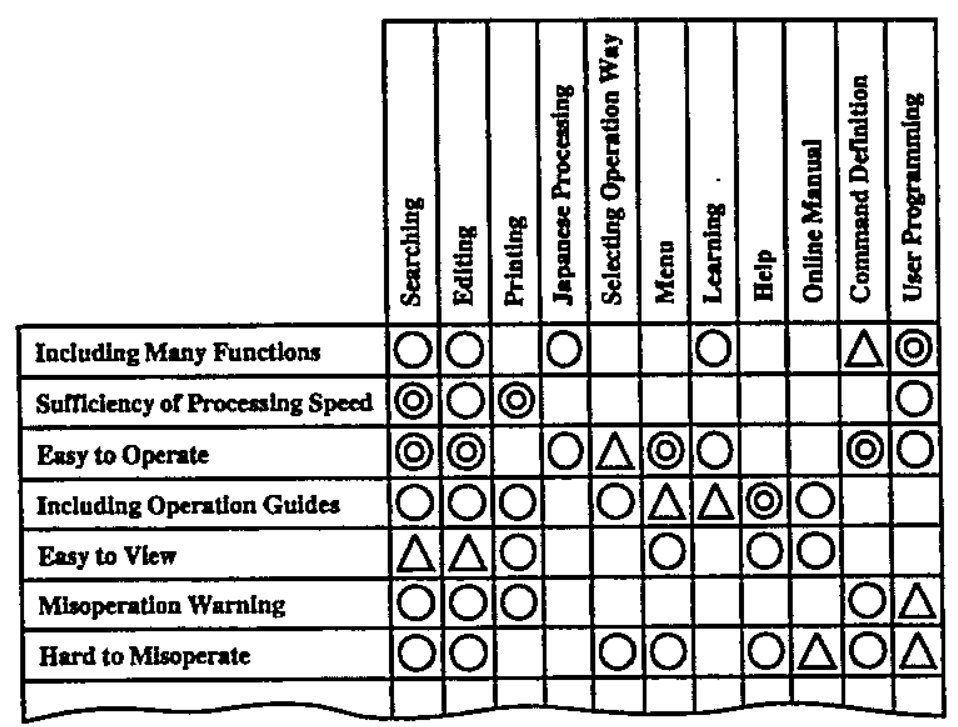

Figure 3: A part of an example of relationship table 
quirement weight vector which is transformed into the developer's side is $v^{t}$. We can formulate the relationship between $u$ and $v^{\prime}$ as follows:

$$
v^{\prime}=W u
$$

where $W$ is a transposed relationship matrix. With this formula, the developer can understand which functions the user wants and their priorities by transforming the user's requirement into the dimension of the developer's requirement. We have to assign the relationship strengths carefully because they will influence on the result straight. For example, we apply the following rule to decide relationship values to reflect the user's requirement as aggressively as possible.

1. To avoid the misunderstanding about the meaning of technical terminologies and about the developer's requirement elements, the developer should explain them to the user.

2. The user checks all of each element of relationship table and fixes their relationship values subjectively. Incidentally, the obscure part of the relationship is fixed after getting the advice of the developer.

3. After that, the developer checks a relationship table.

Then, if there is any contradictory point or any improvement, the developer points out the part to the user and the user corrects it. Therefore, it is desirable that the user and the developer should cooperate together to fix the relationship values.

\section{Inverse Transformation of Requirement Weight Vector}

Next, an inverse transformation technique which make feed back from a developer's requirement to a user is described. The relationship transpose matrix $W$ in formula (5) is rectangular matrix generally. According to the theory of the generalized inverse matrix(Y.Okamoto,1992), it is known that an inverse matrix of $W$ exists uniquely when the condition of Moore-Penrose generalized inverse matrix (shown in the following) is met.

$$
\begin{gathered}
\left(W W^{+}\right)^{t}=W W^{+} \\
\left(W^{+} W\right)^{t}=W^{+} W \\
W W^{+} W=W \\
W^{+} W W^{+}=W^{+}
\end{gathered}
$$

where $W^{+}$is a Moore-Penrose generalized inverse matrix. It is known that an optional rectangular matrix has a Moore-Penrose generalized inverse matrix uniquely. That is, supposing that the developer's requirement. weight vector is $v$, the requirement weight vector $u^{\prime}$ which is transformed into the user's side is shown by formula (10).

$$
u^{\prime}=\dot{W}^{+} v
$$

Therefore, the developer becomes able to grasp the user's viewpoint at the level of his own viewpoint by using formula (5). In the same way, the user becomes able to grasp the developer's viewpoint at the level of his own viewpoint by formula (10). In other words, at the same time as the user's requirement is directly reflected into software functions, the developer's requirement is fed back to the user.

\section{Tradeoff Analysis Among Requirement Weight Vector}

$u$ and $v^{\prime}$ in formula (5) express a user's weight vector and its transformed weight vector at the early stage before starting the consensus making process. In the same way, $v$ and $u^{\prime}$ in formula (10) express a developer's weight vector and its transformed weight vector at the early stage. Generally, the vector direction between $u$ and $u^{\prime}$ (or $v$ and $v^{\prime}$ ) is different in this stage. In order to step up each other for the consensus making, we suppose that the developer changes 
his requirement vector as indicated by $\Delta v$, whereas the user changes his requirement vector as indicated by $\Delta u$. A littte change of $\Delta v$ and $\Delta u$ is recommended. Next, we define the following index functions using the above parameters.

- The index functions shown to the user

$$
\begin{gathered}
S\left(\Delta u, \Delta u^{\prime}\right)=(u-\Delta u)^{t}\left(u^{\prime}-\Delta u^{\prime}\right) \\
R u(\Delta u)=u^{t}(u-\Delta u) \\
R d\left(\Delta u^{\prime}\right)=u^{\prime t}\left(u^{\prime}-\Delta u^{\prime}\right) \\
C=S /(\alpha R u+\beta R d)
\end{gathered}
$$

- The index function shown to the developer

$$
\begin{gathered}
S\left(\Delta v, \Delta v^{\prime}\right)=(v-\Delta v)^{t}\left(v^{\prime}-\Delta v^{\prime}\right) \\
R u\left(\Delta v^{\prime}\right)=v^{\prime t}\left(v^{\prime}-\Delta v^{\prime}\right) \\
R d(\Delta v)=v^{t}(v-\Delta v) \\
C=S /(\alpha R u+\beta R d) \\
\text { where }(\alpha+\beta=1, \alpha>0, \beta>0)
\end{gathered}
$$

where $S$ shows the approaching degree between the modification of the user's sense of value and that of the developer's one, $R u$ shows the changed degree of the user's requirement, $R d$ shows the changed degree of the developer's requirement, and $C$ shows the consensus making degree of both. $R d$ and $R u$ often mean the degree of dissatisfaction or concession. $\alpha$ and $\beta$ are suitable weighting coefficient that is decided by both conditions. Then, the values of formula (11)-(14) are shown to the user on the screen display by graphical charts. Similarly, the values of formula (15)-(18) are shown to the developer. While viewing these values, both the user and the developer change their own requirement weight vectors with interactive mode for the consensus making. These changes are reflected in the above index function's value at once. The consensus making is supported by repeating the above sensitivity analysis.

\section{Example of Implementation}

We are now implementing a prototype system on a SUN work station with $\mathrm{X}$ Window system environment. It has some groupware functions(WYSIWIS:What You See Is What I See). We show a small example of deciding functional specification in software development, in which our method apply to the function analysis process to reflect both a user's requirement and a developer's one. The upper process of software design such as planning, making of specification is essential to develop software. In this process, creative thinking is the main part and is done by the group cooperative work. Problem solving, software design, progresses while both the user and the developer show their own requirements mutually and look for the compromise of their requirements. But actually, communication gap often occurs among them which obstructs their consensus making. Therefore, the improvement of above-mentioned group cooperative work can be expected with our proposed method.

The user's requirement of this example obtained by KJ supporting tool software (i.e. Diagram Abductor) has a nested structure as shown in Figure 4. The user's requirement structure can be derived directly by this nested structure and it is shown in Figure 5. Each value of requirement weight is denoted at the right side of its own requirement element box. Figure 6 denotes an example of the operation screen during a pair comparison is done and the values of requirement weight are calculated. The developer's requirement structure is abbreviated, but it has the similar structure as the user's one. The requirement acquisition resulting in the early stage is shown in Figure 7 . The left upper window denotes the original user's requirement, the left lower window denotes the user's requirement transformed into the dimension of developer's requirement, the right lower window denotes 


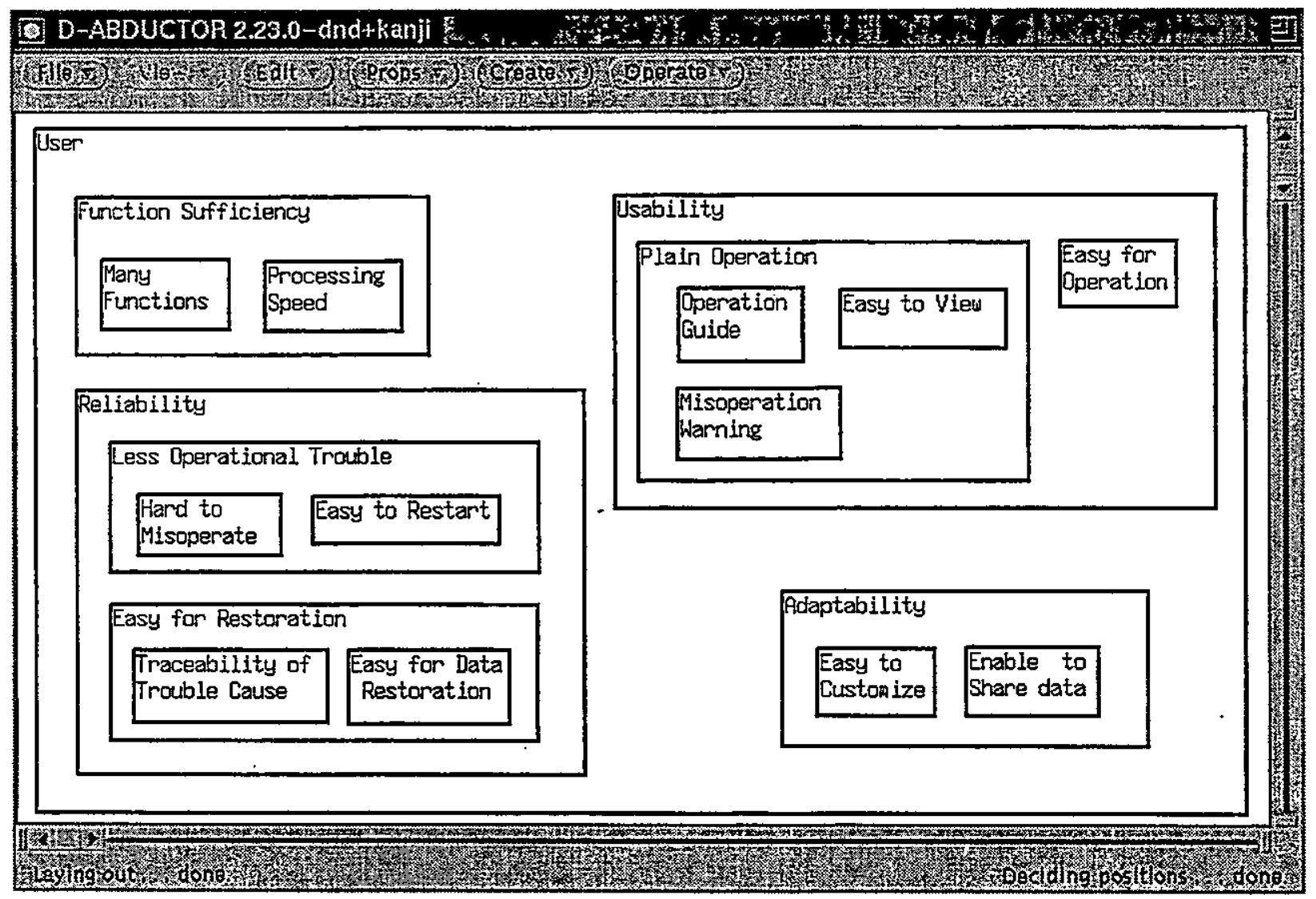

Figure 4: Example $\mathrm{KJ}$ chart of user's requirement

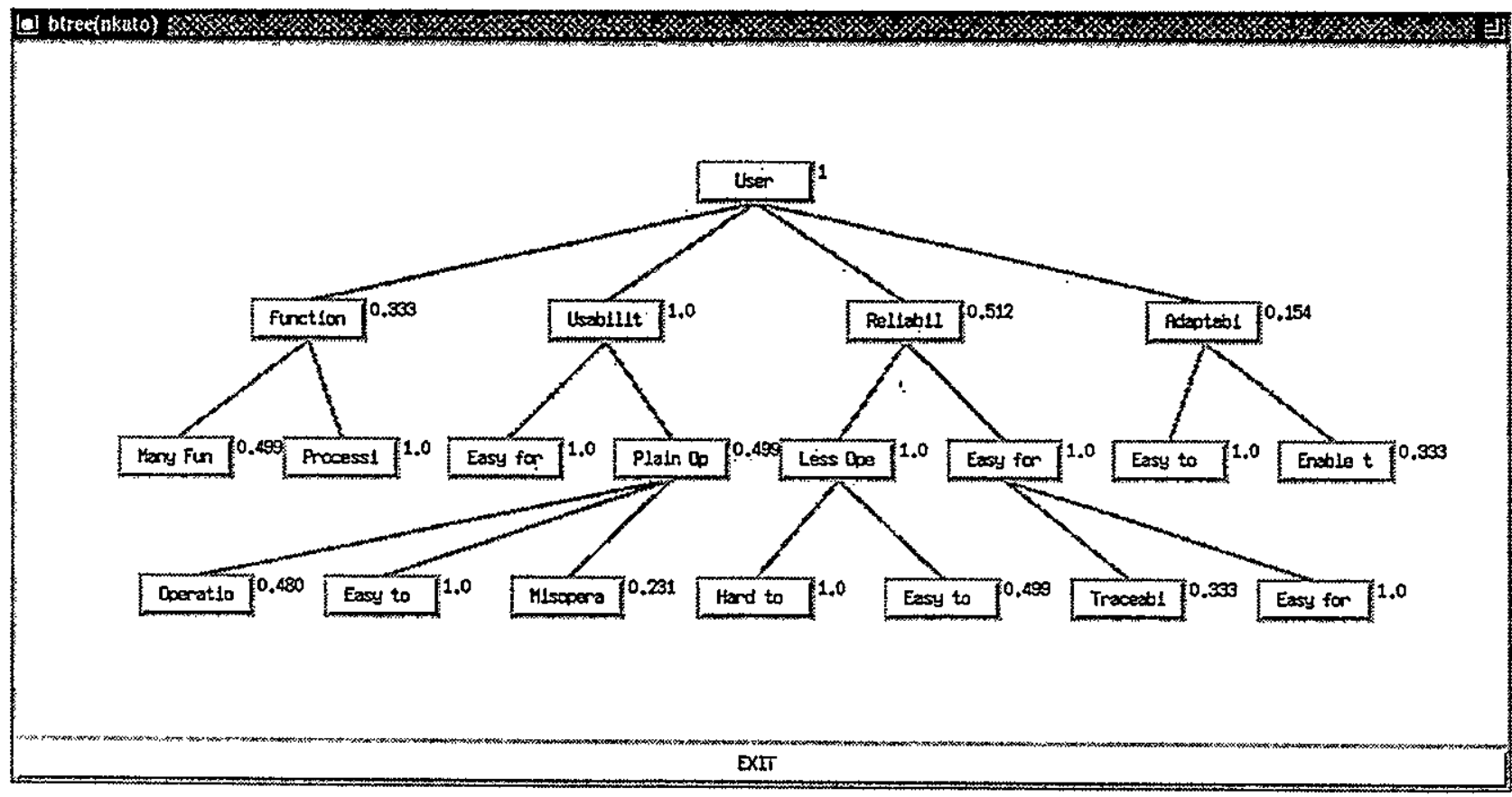

Figure 5: Example of user's requirement structure 


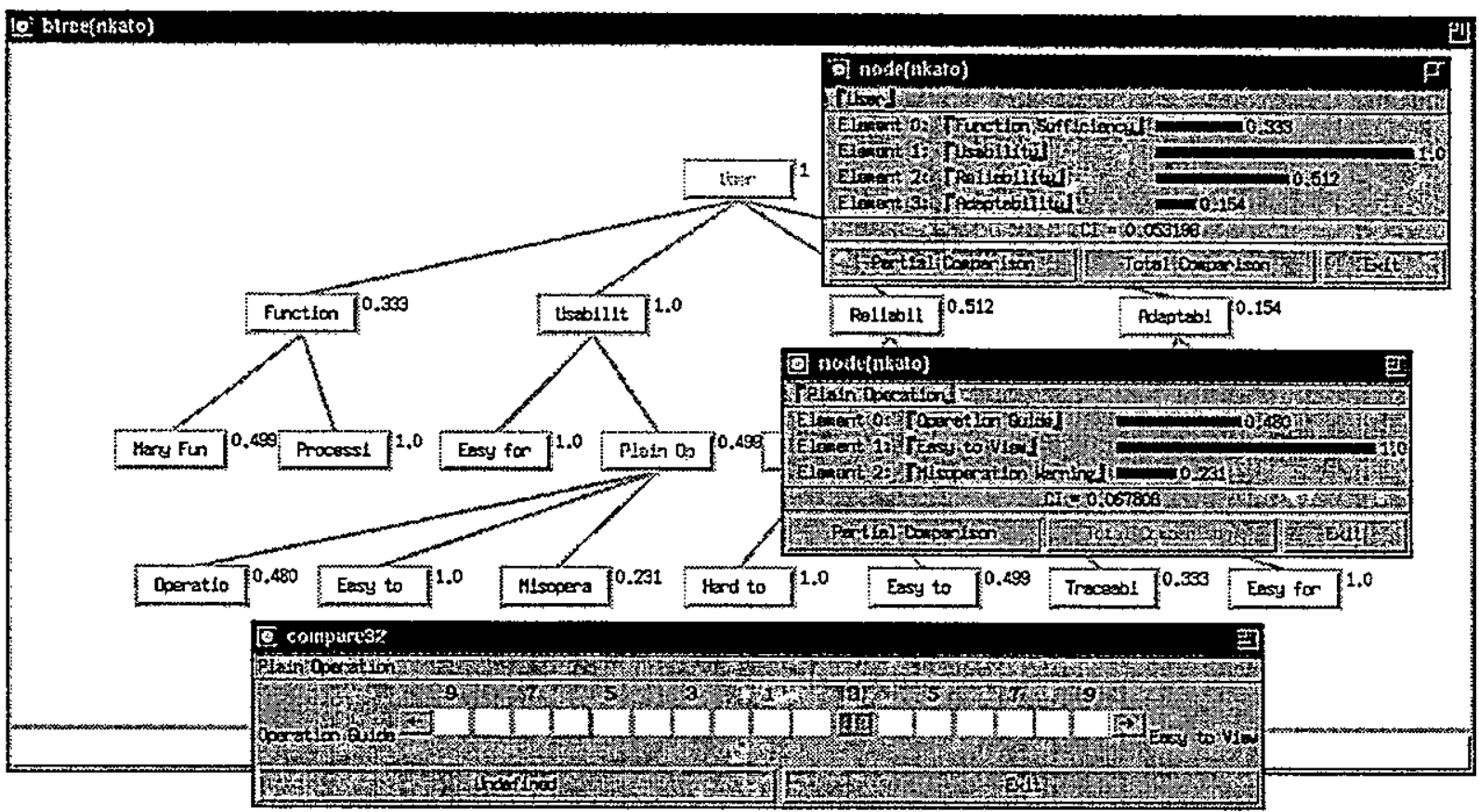

Figure 6: Example of pair comparison and calculation of requirement weight

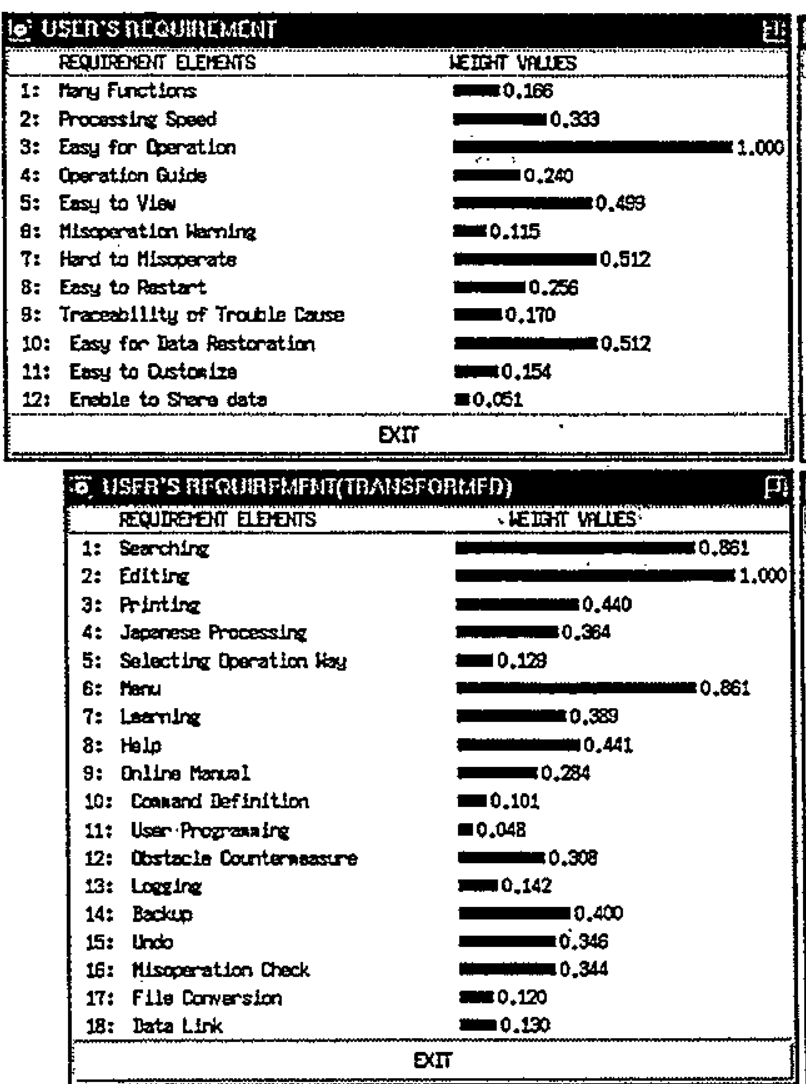

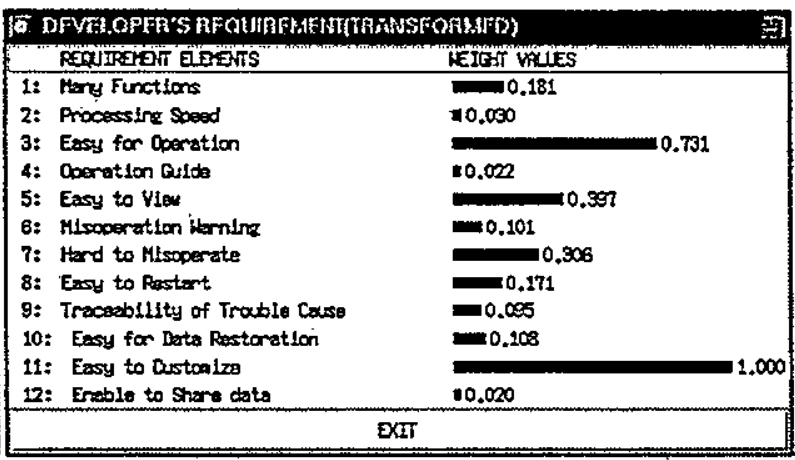

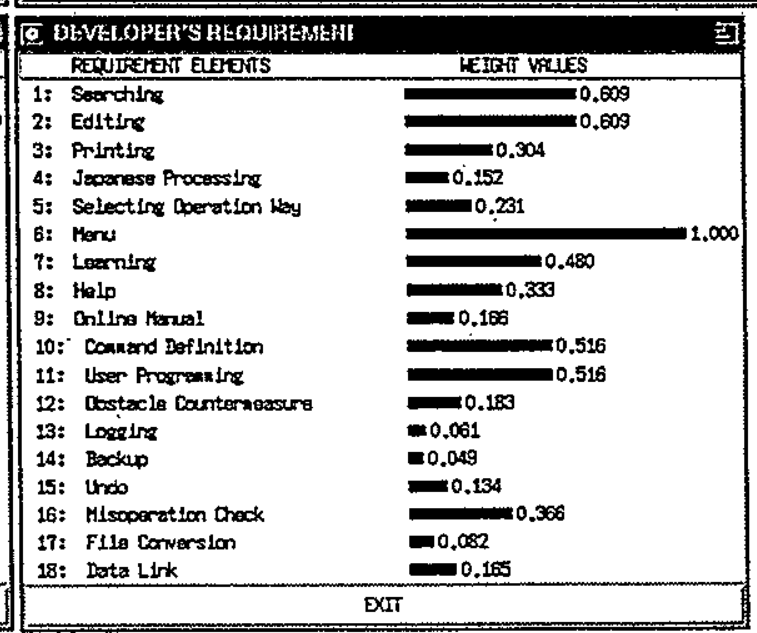

Figure 7: Example of requirement contents in the early stage 


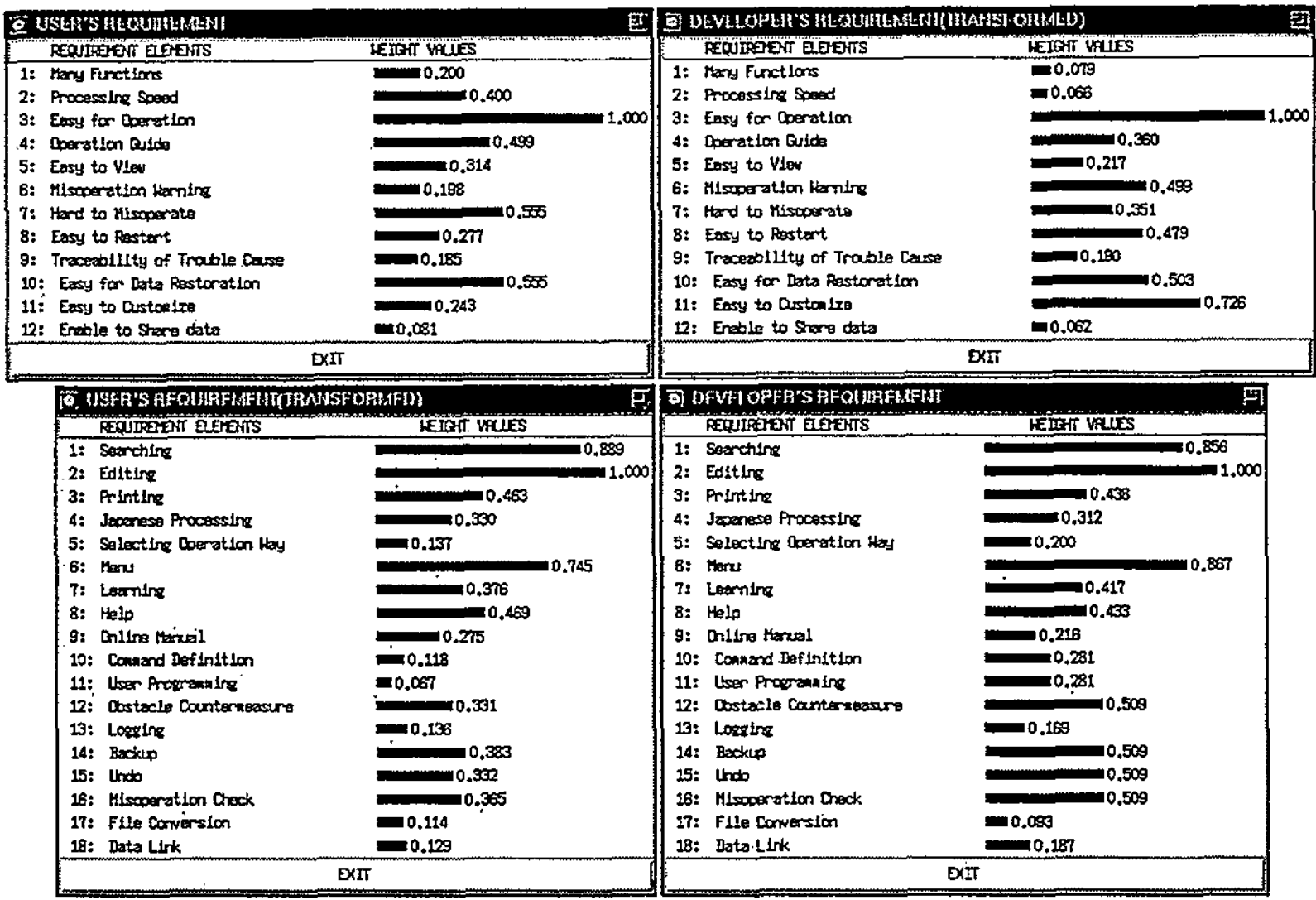

Figure 8: Example of requirement contents in the final stage

the original developer's requirement, and the right upper window denotes the developer's requirement transformed into the dimension of user's requirement.

As a result of the tradeoff analysis operation, the consensus making was achieved to some extent, but it didn't extend to the last satisfied condition. Therefore, their requirement structures have to be reconsidered once again by using our requirement acquisition module. Then, after trying to operate tradeoff analysis once again, the consensus making result was better than the last time. A result at the final consensus making stage is shown in Figure 8. The requirement function analysis of software development which reflects both the user's and the developer's requirements progresses in this way. However, through more future cases, the objective and quantitative evaluation experiment on our system is necessary.

As for qualitative èvaluation, we confirmed some effectiveness of our system.

- Concerning with ease of constructing requirement structure

It is not easy to construct appropriate requirement structure in group decision-making because its task needs expert knowledge and heavy loads. To reduce working loads and to operate intelligibly for even if non-expert user, we provided a method that the whole requirements are classified into subjective and objective requirement elements and each requirement structure is constructed separately by using KJ method and AHP. We confirmed from the above example that our system makes it easier to embody and refine requirement structure gradually and creatively by interactive support fuctions.

- Concerning with equality between participants in consensus making process In conventional method, a user often has dissatisfaction about the result of requirement analysis 
because the user's requirement is not sufficiently reflected to the developer. From the above example, the user's requirement was aggressively reflected to the developer's side and consensus making progressed in the situation which the user and the developer are equal. At this point, our method is different mainly as compared with the conventional requirement analysis which the developer takes a leadership. One of the characteristics of our method is that requirement analysis which a user takes part in the planning directly can also be supported.

\section{Conclusions}

We proposed a new method of consensus making support for group decision problem solving. A characteristic of our method is to integrate divergent thinking support functions and convergent thinking support functions using KJ method, decision-making method, and QDA method. Our method can deal especially with the cooperative work among the perticipants who have different senses of value with each other. Two types of tradeoff analysis mechanism were designed and implemented for consensus making. Through an example for consensus making in case of software development, the way to support both requirement acquisition and function requirement analysis between a user and a developer was described. We provided a distributed environment to show the user and the developer both of their requirements by transforming them into the dimension where they can interpret each other's requirement. In other words, by using our system, the subjective evaluation of the user and the objective evaluation of the developer can be connected mutually. With this, some latent requirement elements can be discoverd newly and the reconsideration of the requirement contents can be done rationally. In this way, their consensus making process can be advanced with the bi-directional repetitive transformation procedure.

\section{References}

H. Ueda and S. Kunifuji(1993), "GRAPE: Knowledge acquisition support groupware for the classificationchoice problem - The design principles, groupware functions, and a suggestion to extend for the planning problem -, R. Zurawski and T. S. Dillon (Editors) in Modern Tools for Manufacturing Systems," Elsevier Science Publishers, 119-135.

J. N. Warfield(1974), "Developing Interconnection Matrices in Structural Modeling," IEEE Trans. of SMC, Vol.SMC-4, No.1:

S. Kunifuji and T. Takeshima(1979), "Reachability Analysis, on a Directed Graph with Compound Vertices - an Extension of ISM -,". Technical Report of the IEICE CAS79-110,61-66 (in Japanese).

L. A. Zadeh(1971), "Similarity Relations and Fuzzy Ordering," Infor. Sciences, Vol.3, 177-200.

T. L. Saaty(1980), The Analytic Hierarchy Process, McGraw-Hill.

H. Nagata(1994), "A Study on Decision Making Method that Enforces the Decision Maker to Judge with Rationality," Master's Thesis of School of Information Science of JAIST (in Japanese).

J. Kawakita(1975), The KJ Method : A scientific approach to problem solving, Kawakita Research Institute.

N. Takeda, A. Shiomi, K. Kawai, H. Ohiwa(1993), "Requirement Arialysis by KJ Editor," Proc. RE'93, IEEE Int. Symp. on Requirement Engineering 1993, San Diego California, USA.

H. Ohiwa, K. Kawai, M. Koyama(1990), "Idea Processor and KJ method," J. Information Processing, Vol.13; 44-48. 
K. Sugiyama and K. Misue(1991), "Visualization of Structural Information; Automatic Drawing of Compound Digraphs," IEEE Trans. of SMC, Vol.21, No. 4,876-892.

N. Kato, S. Kunifuji(1995), "A Study of Function Analysis Support Reflecting User's Requirement for Software Development," Information Processing Society of Japan SIG Notes 95-GW-9, 63-68(in Japanese).

S. Kunifuji, T. Tamura, and N. Kato(1995), "A Study of Consensus Making Support System based on a Relationship Matrix," The 17th Meeting on System Engineerong of the SICE, 29-36(in Japanese).

A. Ohmori(1994), "Software Quality Deployment Approach: Framework Design, Methodology and Example," Software Quality Journal 3, 209-240.

Harker, P. T.(1987), "Incomplete Pairwise Comparisons Method in The Analytic Hierarchy Process," Màth. Modeling, 9, 353-360.

H. Ichihashi and H. Tanaka(1987), "AHP using non-additive weight," Operations Research Society of Japan, Vol.1987, 215-216 (in Japanese).

T. Masuda(1987), "Sensitivity Coefficients of Consistency Index and Priorities Used in the Analytic Hierarchy Process," The Transactions of The Institute of Electronics, Information, Communication Engineers, A Vol.J70-A, No.11, 1562-1567 (in Japanese).

Y. Okamoto(1992), Inverse Problem and its Solving Method, Ohmsha(in Japanese). 